\title{
The spectrum of small-bowel lesions of AL-type amyloidosis at capsule endoscopy
}

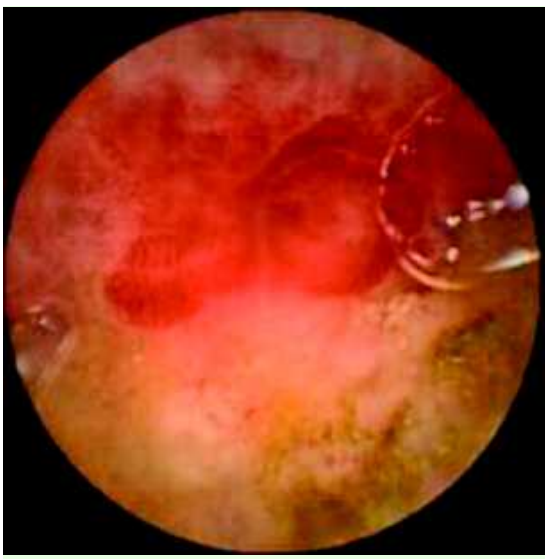

Fig. 1 Patchy red plaque lesion consistent with mucosal hematoma.

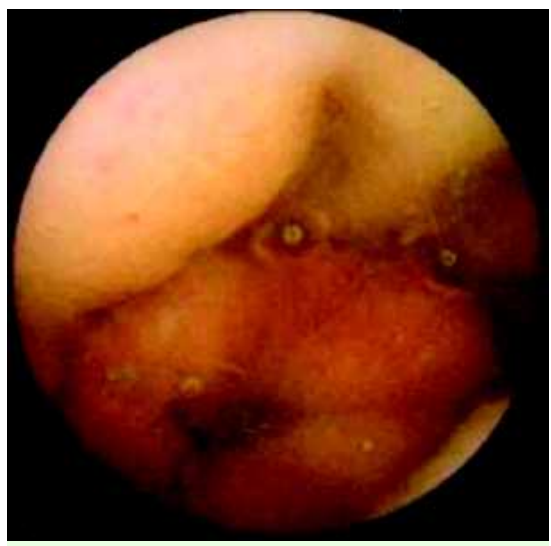

Fig. 2 Thickened folds causing intraluminal stenosis.

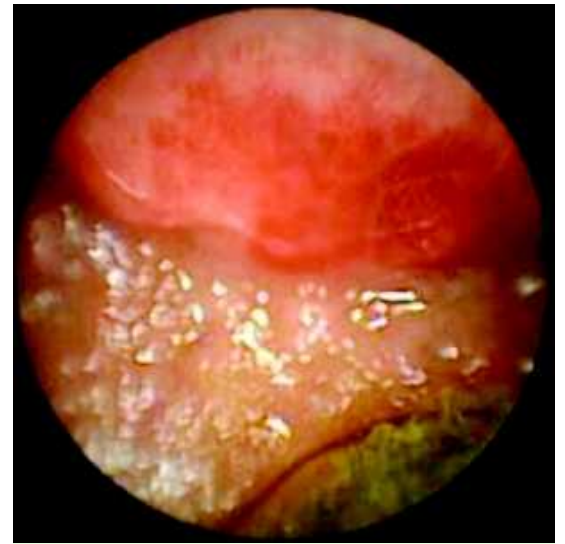

Fig. 3 Irregular-shaped ulcerations.

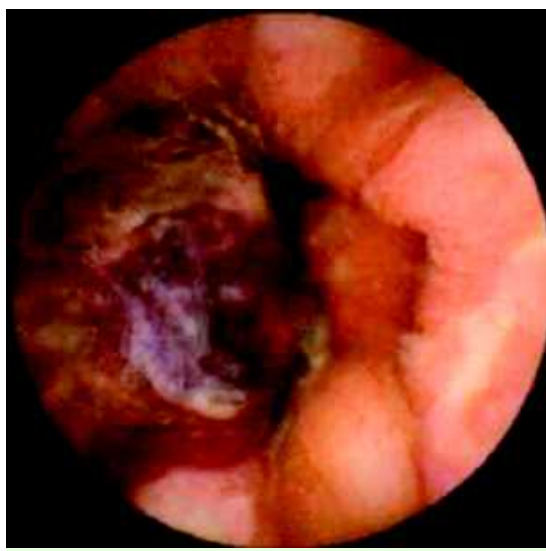

Fig. 4 Actively bleeding polypoid mass with visible vessel.
A 49-year-old man presented at the emergency department because of sudden onset of epigastric pain and melena. Blood tests showed a slight increase in cardiac troponin I, and electrocardiogram revealed low $Q R S$ voltages with no abnormality consistent with acute myocardial injury. At echocardiography, an initial diastolic dysfunction and left ventricular wall thickening with a sparkling appearance of the myocardium were found. An urgent upper gastrointestinal endoscopy showed an active bleeding Dieulafoy's lesion in the duodenal bulb, which was successfully treated with epinephrine injection and argon plasma coagulation; two clean-based irregular ulcers in the second tract of the duodenum were also found. The clinical course was characterized by the recurrence of episodes of massive hematochezia and melena, requiring multiple blood transfusions. Colonoscopy and upper endoscopy results were nondiagnostic, and thus capsule endoscopy was performed. It showed various findings throughout the entire small bowel, such as multiple patchy red plaque lesions (mucosal hematoma, Fig. 1), thickened folds causing, in some instances, intraluminal stenosis ( $\bullet$ Fig. 2), irregular-shaped ulcerations ( Fig. 3), and polypoid protrusions or masses, some of which had ulcerations and visible vessels ( $\bullet$ Fig. 4).

Histopathologic evaluation of biopsy specimens from the stomach, duodenum, and proximal jejunum revealed a massive deposition of amorphous eosinophilic material in the lamina propria and muscularis mucosae. Congo red stain was positive and demonstrated green birefringence under polarizing microscopy 


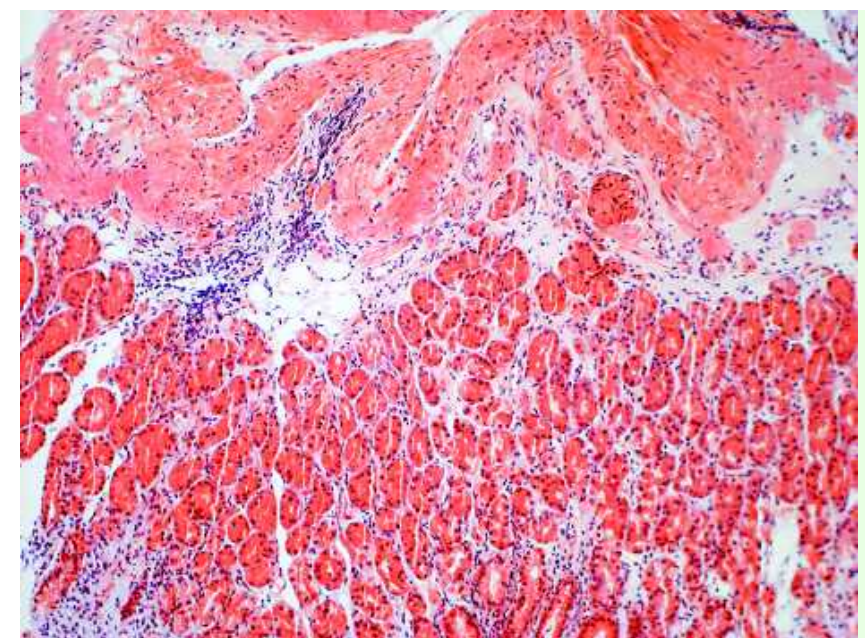

Fig. 5 Histologic examination of a gastric biopsy showing amorphous material extensively involving mucosa and submucosa (original magnification $x$ 200).
( Fig. 5). Based on bone marrow aspiration, the presence of k-type light monoclonal chains in the serum and urine and the demonstration of Bence Jones protein in the urine led to the final diagnosis of primary systemic AL amyloidosis with prominent involvement of the gastrointestinal tract and myocardium.

Endoscopy_UCTN_Code_CCL_1AC_2AH

G. Mandelli ${ }^{1}$, F. Radaelli ${ }^{1}$, A. Amato ${ }^{1}$, N. Terreni ${ }^{1}$, S. Paggi ${ }^{1}$, G. Spinzi ${ }^{1}$,

E. Ceretti ${ }^{2}$, V. Terruzzi ${ }^{1}$

1 Department of Gastroenterology, Valduce Hospital, Como, Italy

2 Department of Pathology, Valduce Hospital, Como, Italy

Bibliography

DOI $10.1055 / \mathrm{s}-2007-995543$

Endoscopy 2009; 41: E51 - E52

(c) Georg Thieme Verlag KG Stuttgart · New York · ISSN 0013-726X

Corresponding author

F. Radaelli MD

Department of Gastroenterology

Valduce Hospital

Via Dante 11

22100 Como

Italy

Fax: +39-31-308047

francoradaelli@virgilio.it 